\title{
Complexity of VTA DA neural activities in response to PFC transection in nicotine treated rats
}

Ting Y Chen, Die Zhang, Andrei Dragomir, Yasemin M Akay, Metin Akay*

\begin{abstract}
Background: The dopaminergic (DA) neurons in the ventral tegmental area (VTA) are widely implicated in the addiction and natural reward circuitry of the brain. These neurons project to several areas of the brain, including prefrontal cortex (PFC), nucleus accubens (NAc) and amygdala. The functional coupling between PFC and VTA has been demonstrated, but little is known about how PFC mediates nicotinic modulation in VTA DA neurons. The objectives of this study were to investigate the effect of acute nicotine exposure on the VTA DA neuronal firing and to understand how the disruption of communication from PFC affects the firing patterns of VTA DA neurons.

Methods: Extracellular single-unit recordings were performed on Sprague-Dawley rats and nicotine was administered after stable recording was established as baseline. In order to test how input from PFC affects the VTA DA neuronal firing, bilateral transections were made immediate caudal to PFC to mechanically delete the interaction between VTA and PFC.

Results: The complexity of the recorded neural firing was subsequently assessed using a method based on the Lempel-Ziv estimator. The results were compared with those obtained when computing the entropy of neural firing. Exposure to nicotine triggered a significant increase in VTA DA neurons firing complexity when communication between PFC and VTA was present, while transection obliterated the effect of nicotine. Similar results were obtained when entropy values were estimated.

Conclusions: Our findings suggest that PFC plays a vital role in mediating VTA activity. We speculate that increased firing complexity with acute nicotine administration in PFC intact subjects is due to the close functional coupling between PFC and VTA. This hypothesis is supported by the fact that deletion of PFC results in minor alterations of VTA DA neural firing when nicotine is acutely administered.
\end{abstract}

\section{Background}

The mesocorticolimbic dopamine system, consisting of the ventral tegmental area (VTA), prefrontal cortex (PFC) and nucleus accumbens (NAc), is a critical substrate for the neural adaptations that underlie addiction [1]. The dopamine (DA) neurons in VTA and their projection areas, including PFC, NAc, and amygdala, are thought to be very important in the reward driven behavior induced process by the drugs of addiction [1-5]. Nicotine is a biologically active substance that promotes tobacco use and has caused the global population health

\footnotetext{
* Correspondence: makay@uh.edu

Department of Biomedical Engineering, Cullen College of Engineering, University of Houston, Houston, TX 77204, USA
}

and economical problems. Unfortunately, nicotine dependence creates problems for smokers to quit. The mesocorticolimbic dopamine pathways have been shown to be stimulated by nicotine. The stimulation originates from VTA and resulting in DA secretion within the NAc and PFC is essential for the reinforcing effects of nicotine [6]. Moreover, other neurotransmitter pathways like glutamatergic neurons projecting from PFC to VTA are also involved in the motivational effects of nicotine $[7,8]$. The important role played by glutamatergic pathways in excitation of mesocorticolimbic dopaminergic neurons by nicotine has been demonstrated by many previous studies [9].

The firing activities of VTA DA neurons and addictive behavior of the animals are believed to be controlled by

\section{() Biomed Central}


the glutamatergic synaptic inputs from PFC [10-14]. The PFC is a key structure for executive functions of the brain $[15,16]$, and has been shown to regulate the firing pattern of VTA DA neurons. Therefore, the burst firing in VTA DA neurons increases with PFC stimulation and the opposite effect is shown with PFC inactivation [17-21]. The strengthening of input from PFC to VTA plays an important role in the behavioral sensitization development, a well-known model for addiction [22-24]. Evidence has shown the functional input loss from PFC and/or NAc may reduce the effects of these drugs on the addiction process [13,25-27]. Studies have demonstrated that under in vivo conditions, the VTA DA neurons produce single spikes and/or burst firing. Additionally, they are capable of firing in a slow oscillatory $(\mathrm{SO})$ pattern. The $\mathrm{SO}$ generation needs inputs from other brain area (i.e. PFC) $[28,29]$.

Previous studies show that systemic nicotine injection can increase the firing rate and percentage of bursting firing of VTA DA neurons [30-33]. However, the PFC transection only excited $28 \%$ of the VTA DA neurons which could be stimulated only by systemic nicotine activation, but not by the PFC [32,33]. Also, we have known that VTA DA neurons' bursting firing mode needs excitatory inputs. Therefore, we hypothesize that systemic exposure to nicotine significantly affects the complexity of firing of the VTA DA neuron and this alteration should be based on the intact input from other brain areas. Since PFC is the main source of excitatory inputs to the VTA, the effect of nicotine on the complexity of VTA DA neuronal firing will be reduced, when the pathway between PFC and VTA is disconnected. To test this hypothesis, we recorded VTA DA neurons firing and analyzed the data using the advanced nonlinear dynamical analysis method based on the Lempel-Ziv (LZ) estimator.

Traditional analysis methods of neuronal firing activity consist only in measuring spike amplitude and/or extracting spike frequency information in order to characterize the changes produced in the VTA or other brain areas by different physiological factors or pharmacological treatments [34,35]. However, the use of such methods often renders comparisons within subject groups not possible. The amplitude characteristics or frequency of rhythms may differ from subject to subject. Additionally, they may not offer any insight on the firing patterns generated by the neural activity. Therefore, more robust and meaningful analysis methods need to be used for the dynamical analysis of neural recordings. The dynamical analysis is especially relevant in the context of VTA DA neurons, which are part of neural networks that receive inputs from several other brain areas. Therefore, in this study, we have analyzed the dynamics (complexity) of nicotine-induced neuronal firing pattern in the VTA DA neurons in both PFC intact and transected Sprague Dawley (SD) rats using the Lempel-Ziv (LZ) method. We also estimated the entropy values of the recorded firing activity and compared the results obtained from LZ analysis and entropy [36].

\section{Methods}

\section{Electrophysiological recordings}

All experimental protocols and surgeries were approved by The Institutional Animal Care and Use Committee of Arizona State University. We used male SpragueDawley (SD) rats from Charles River Laboratories (Wilmington, MA) weighting between 250 and 300 grams. All animals were anesthetized with chloral hydrate (400 mg/kg, intraperitoneal (i.p.) injected) and mounted with stereotaxic apparatus (Narishige, Japan) for extracellular single-unit recording. The extracellular recording pipette was filled with $2 \mathrm{M} \mathrm{NaCl}$ (Sigma) and $0.5 \%$ Chicago sky blue (Sigma) solution and placed into the VTA through a small burr hole in the skull (2.7-3.3 mm anterior to the lambda and 0.5-0.9 mm lateral to the midline) by an electro-microdriver. DA neurons, usually at $6.5-8.5 \mathrm{~mm}$ below the cortical surface, were identified according to the well established electrophysiological criteria [37-41]. After stable recording was established for a minimum of five minutes as baseline, (-) nicotine hydrogen tartrate salt (Sigma Chemical Co., St. Louis, $\mathrm{MO})$ at a smoking-relevant concentration $(0.5 \mathrm{mg} / \mathrm{kg}$, i.v. via tail vein) was administered and recordings were continued for at least 15 minutes. Mereu et al [42] studied the influence of various doses of nicotine on Dopamine (DA) neurons in rats either general or local anesthesia. Their results showed the optimal dose of nicotine $(0.5 \mathrm{mg} / \mathrm{kg})$ that produced a significant increase in the firing rate of DA neurons. Stolerman et al performed similar studies [43] that confirmed that $0.5 \mathrm{mg} /$ $\mathrm{kg}$ was an optimal and effective dose to study the influence of nicotine in the neural firings of DA neurons. Many others [31,44,45] also used $0.5 \mathrm{mg} / \mathrm{kg}$ dose of nicotine to study the behavior of DA neurons. Therefore, these studies encouraged us to focus on the single, optimal dose to investigate the influence of nicotine on the dynamics of neural firings of DA neurons. The body temperature was maintained at 36 to $38^{\circ} \mathrm{C}$. The recording sites were marked by ejection of Chicago sky blue and examined using standard histology methods at the end of experiments $[32,33,40]$.

\section{PFC transection}

To study the interaction of PFC inputs to the VTA DA neurons, bilateral transections were made immediate caudal to the PFC to disrupt the communication between PFC and VTA DA neuron. A slit was drilled in the skull $2.0 \mathrm{~mm}$ anterior to bregma. Without damaging 
the main artery, a sharp blade was lowered to the base of skull, to completely interrupt the connections between the PFC and the rest of the brain. All surgical procedures were done under anesthetized condition $[32,33,40]$.

\section{Data acquisition and analysis}

The firing activities of VTA DA neurons were recorded from five SD rats for both PFC intact and PFC transected rats. Data was acquired and recorded on the same data acquisition system (Powerlab, ADInstruments). We quantified the neural dynamics using the LZ complexity estimator as detailed below. Two-minute segment of data before the injection of nicotine was analyzed with LZ complexity method. After firing rate of DA neuron has reached stable condition in response to nicotine, two minutes of data with the effect of nicotine was analyzed with LZ complexity to understand the dynamics (complexity) of neural firing in response to nicotine exposure to VTA DA neurons with and without input from PFC. All values are expressed as mean \pm SEM. Statistical significance was assessed using paired two-tailed Student's $t$ - tests.

\section{Lempel-Ziv Complexity}

The firing activity recorded from VTA DA neurons arises from complex feedback networks and nonlinear interconnections, which are characteristic for such neural systems. Therefore, we used the LZ estimator as a measure of complexity (regularity) of the firing activities recorded from VTA DA neurons [46-49]. LZ complexity is closely related to information-theoretical methods such as entropy [48] and is able to cope with discrete-time symbolic sequences. It quantifies the rate of new pattern generation along given sequences of symbols. The symbolic representations of time series are particularly favored when low-amplitude noise hampers the data [49].

Therefore, we transformed the neural signals into a finite sequence in the symbolic space. Each sample in the time domain was assigned a symbol, and the total number of unique symbols formed the alphabet of the sequence. Since the data was composed of a series of action potentials that form the response of the neurons to the input, we used a binary alphabet. The time axis was divided into discrete bins. The action potentials were detected using an amplitude threshold, and each time the threshold was crossed, we placed a "1" in the respective bin of the symbolic representation of our signals. All bins with values below the threshold were assigned a "0" [49].

Formally, our signal $x(n)$ was converted into a binary sequence $S=s(1), s(2), \ldots, s(n)$, where

$$
s(i)= \begin{cases}0, & \text { if } \quad x(i)<T \\ 1, & \text { otherwise, }\end{cases}
$$

where $T$ is the threshold and can be chosen as $2 \mathrm{SD}(x$ $(n))$, where $\operatorname{SD}(x(n))$ represents the standard deviation of the original signal $x(n)$ [49].

For computing the LZ complexity, the sequence $S$ is parsed from left to right, and a complexity counter $c(n)$ is increased each time a new subsequence (distinct word) is encountered. The algorithm followed is:

- Let $S(i, j)$ denote a substring of $S$ that starts at position $i$ and ends at position $j$, where $i<j . S(i, j)=$ $s_{i} s_{i+1} \ldots s_{j}$ and when $i>j, S(i, j)=\{\}$. The vocabulary of the sequence $S, V(S)$, is the set of all unique substrings (words) $S(i, j)$ of $S$.

- The parsing procedure starts by comparing a substring $S(i, j)$ to the vocabulary that is comprised of all substrings of $S$ up to $j-1$, that is $V(S(1, j-1))$. If $S(i, j)$ is present in $V(S(1, j-1))$ then update $S(i, j)$ and $V(S(1, j-1))$ to $S(i, j+1) V(S(1, j))$, respectively, and repeat the previous check. If the substring is not present, place a dot after $S(j)$ to indicate the end of a new component, update $S(i, j)$ and $V(S(1, j-1))$ to $S$ $(j+1, j+1)$ and $V(S(1, j))$, respectively, and the process continues. The whole parsing operation begins at $S(1,1)$ and continues until $j=n$, the total length of the binary sequence [47].

For example, the sequence $S=1011110100010$ is parsed as 1.0.11.110.100.010. Therefore, the vocabulary of $S$ is six. Similarly, a sequence $S=$ 0001101001000101 would be parsed as 0.001 .10 .100 . 1000 . 101, and hence yields a vocabulary sized six [46].

LZ complexity is defined as the total number of words in the decomposition, $c(n)$. The normalized LZ complexity is defined as

$$
C_{L Z}=\frac{c(n)}{n / \log _{2} n} .
$$

More details on the LZ method and its implementation are given elsewhere [46-50].

\section{Entropy}

In addition to the LZ estimator, we also analyzed the same data set using the approximated entropy (complexity) since it has been widely used for the analysis of biomedical signals. The entropy estimates can be computed as follows [36]:

$$
H=-\sum_{n} p(n) \cdot \log _{2} p(n)
$$

Where $p(n)$ is the probability of observing $n$ spikes in the time window. The time resolution was $10 \mathrm{~ms}$ and entropy was computed on segments of $20 \mathrm{~s}$ length. 


\section{Results}

To evaluate the firing pattern changes of VTA DA neurons to systemic nicotine exposure, the extracellular single-unit recordings were performed in DA neurons in anesthetized rats as described in methods section. Two minutes of data was divided in 20-second windows for analysis purposes. LZ complexity was estimated for each 20 -second window and the values were averaged. The same procedure was applied for segments before and after nicotine exposure. The data analyzed for nicotine effect was taken after firing rate of DA neuron has reached stable condition in response to nicotine administration.

Figure 1 shows an example of 20-second segment action potential recorded from PFC intact VTA DA neuron before and after nicotine injection. Figure 2 shows an example of 20-second segment action potential recorded from PFC transected VTA DA neuron before and after nicotine injection. Both firing rate and firing pattern look similar when observed with naked eye. The left panel of Figure 3 shows the averaged LZ complexity values from five PFC intact SD rats before and after nicotine administration. The right panel of Figure 3 shows the averaged LZ complexity values from five PFC transected SD rats before and after nicotine administration.
The LZ complexity values were $0.2079 \pm 0.0075$ before nicotine administration and were $0.2454 \pm 0.0067$ after nicotine administration for SD rats with PFC intact. As shown in Figure 3, there is significant increase in the complexity values in DA neurons after nicotine exposure $(p<0.01)$ for PFC intact rats. Figure 3 indicates that nicotine plays an important role in affecting the firing of DA neurons in VTA. Considering that the excitatory input to VTA DA neurons is mainly originated from the PFC, the above results suggests a possibility that systemic nicotine-induced changes of VTA neuron firing might be mediated through an alteration in PFC neural function. To test this hypothesis, we interrupted the PFC and VTA interaction by acute PFC transection. The transection was done mechanically immediate caudal to the PFC by acute transecting both sides of PFC as described in methods. The LZ complexity values were $0.2273 \pm 0.0099$ before nicotine administration and were $0.2248 \pm 0.0101$ after nicotine administration for SD rats with PFC transected. As shown in Figure 3, there is no significant difference $(p=0.8085)$.

In addition to LZ complexity analysis method, we also calculated entropy estimates of the same neural recordings for comparison purposes. The entropy values were 0.2179 \pm 0.0078 before nicotine administration and were $0.2766 \pm$ 0.0100 after nicotine administration for SD rats with PFC

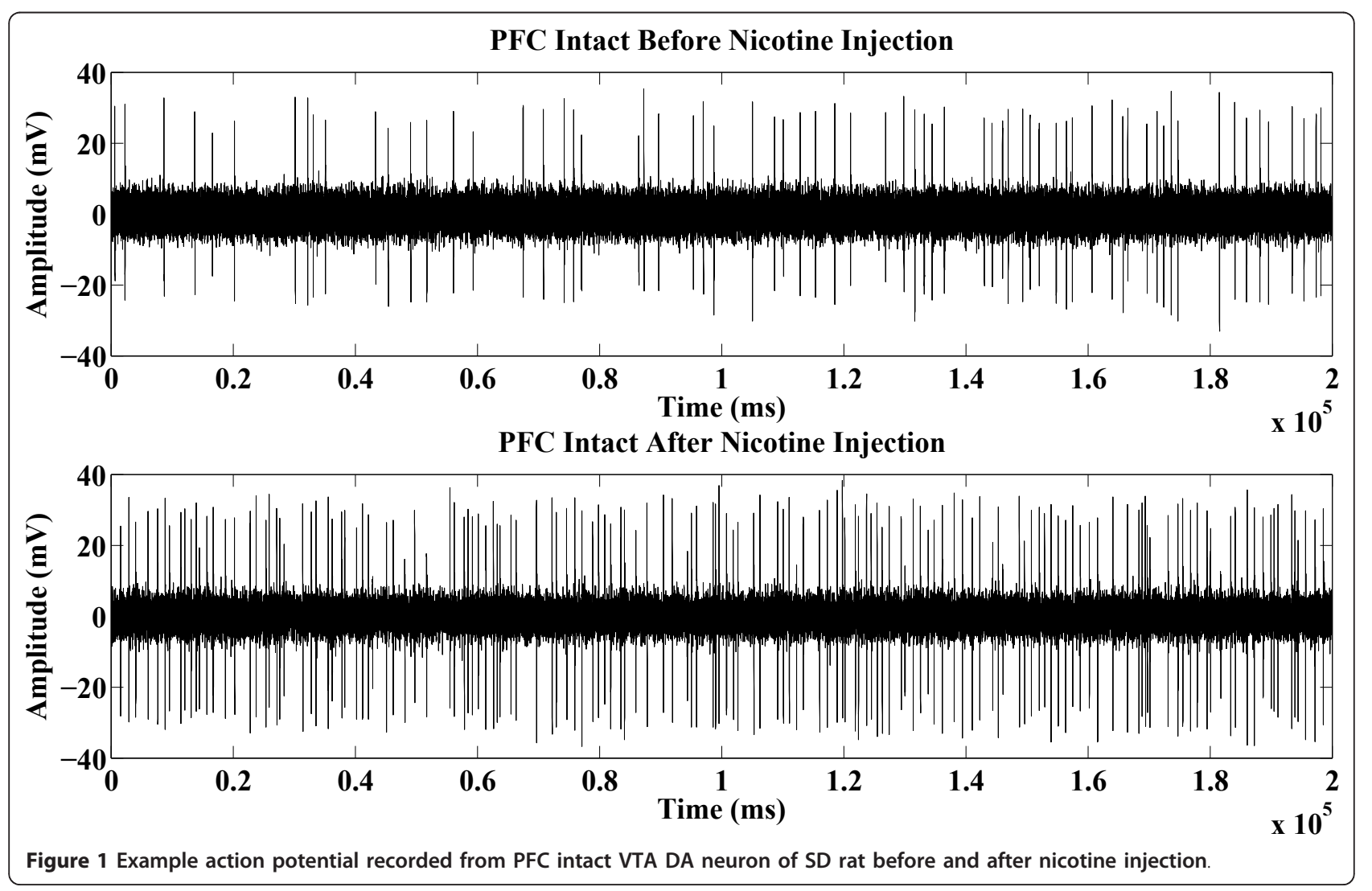



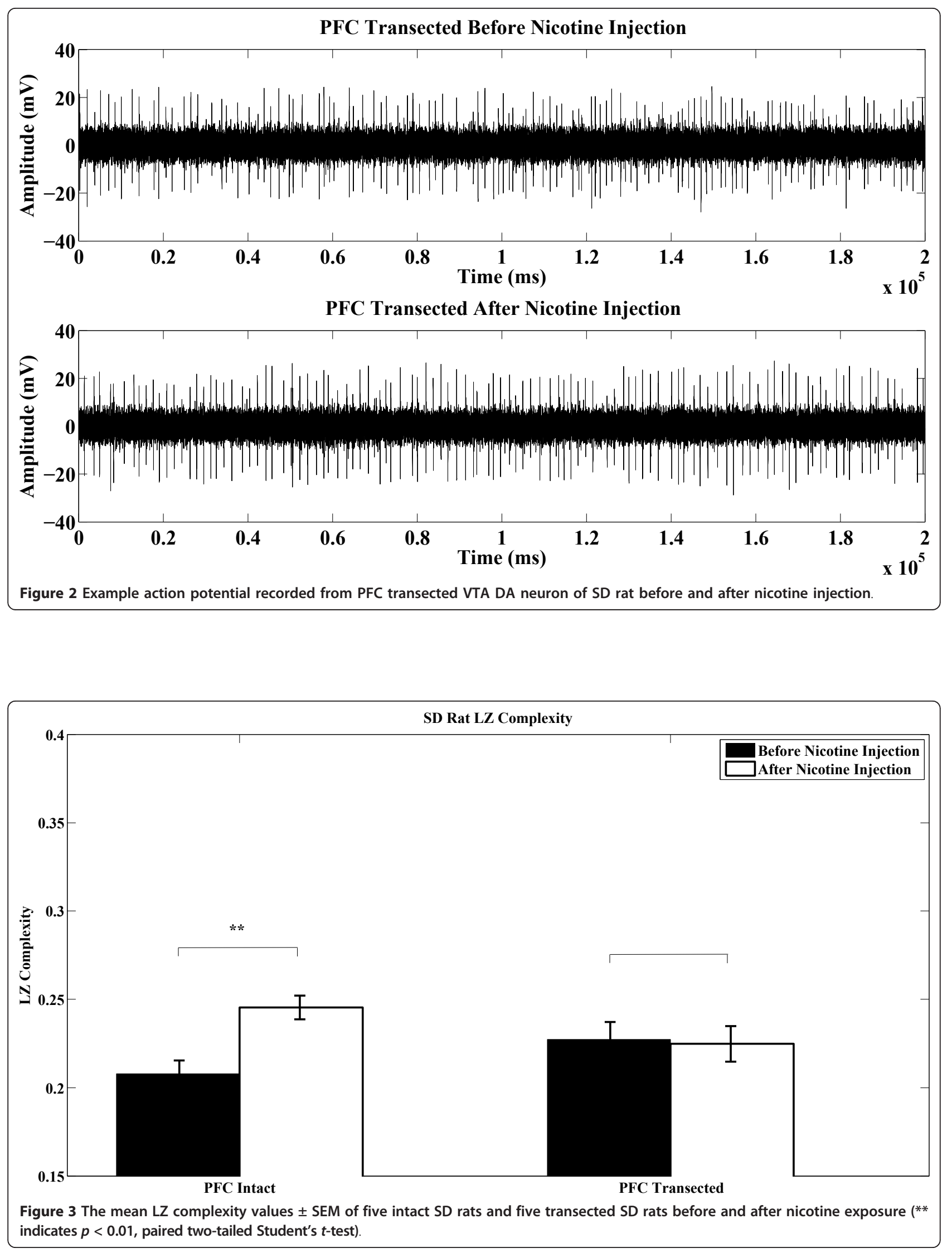
intact. As shown in Figure 4, there is a significant increase in the entropy values in DA neurons after nicotine exposure $(p<0.01)$ for PFC intact rats. The entropy values were $0.2382 \pm 0.0107$ before nicotine administration and were $0.2396 \pm 0.0118$ after nicotine administration for SD rats with PFC transected. As shown in Figure 4, there is no significant difference $(p=0.9319)$.

\section{Discussion and conclusion}

In this study, we used nonlinear dynamical analysis methods based on the LZ method and the approximated entropy to analyze VTA DA neuronal firing activity induced by systemic administration of nicotine on PFC intact and transected rats. The analyses allow us to quantitatively distinguish the firing patterns dynamics of VTA DA action potentials. These patterns may reflect different status of neuronal network synchronization. Nonlinear dynamical analysis of neural patterns demonstrated that nicotine only significantly affects PFC intact rats and this may be due to the close connection between PFC and VTA.

The neural activity recorded from VTA DA neurons arises from complex networks and non-linear interconnections, which are neural systems characteristics. The fact that the neural activity arises from such complex systems, as well as the symbolic-like features of the recorded data, make the use of the LZ complexity measure suitable in the context of the present work [51-68].

Provided by its robustness over other complexity/ entropy measures, the LZ complexity has been applied extensively in biomedical signal analysis as a metric to estimate the complexity of discrete-time physiologic signal. For example, LZ has been used for recognition of structural regularities [54], for complexity characterization of DNA sequences [57-59], to develop new methods for discovering patterns in DNA sequences by applying it to genomic sequences of Plasmodium falciparum [59], and to estimate the entropy of neural discharges (spike trains) $[48,60]$. LZ complexity has also been used to study brain function [62], brain information transmission [63], EEG complexity in patients with Alzheimer's disease [64], epileptic seizures [65], ECG dynamics [66], and to evaluate the nature and dynamics of hippocampal neuronal oscillations [50,69,70].

In recent studies the performance of the LZ estimator was compared to other entropy measures for the analysis of the biomedical signals $[49,71]$. Although LZ complexity was shown to be related to entropy $[48,68]$, it proved to be less sensitive to the length of data [71]. Its better performance in terms of sensitivity to signal bandwidth changes was also reported, when compared to Shannon entropy [71].

All these previous studies encouraged us to use this nonlinear dynamical analysis method, based on the LZ complexity method, to gain insights into the VTA DA neuronal activity induced by systemic administration of nicotine to both PFC intact and transected subjects. The results obtained when using the LZ estimator were confirmed by those obtained when the entropy of the neuronal firing was estimated. Therefore, our results confirm our hypothesis that nicotine significantly affects

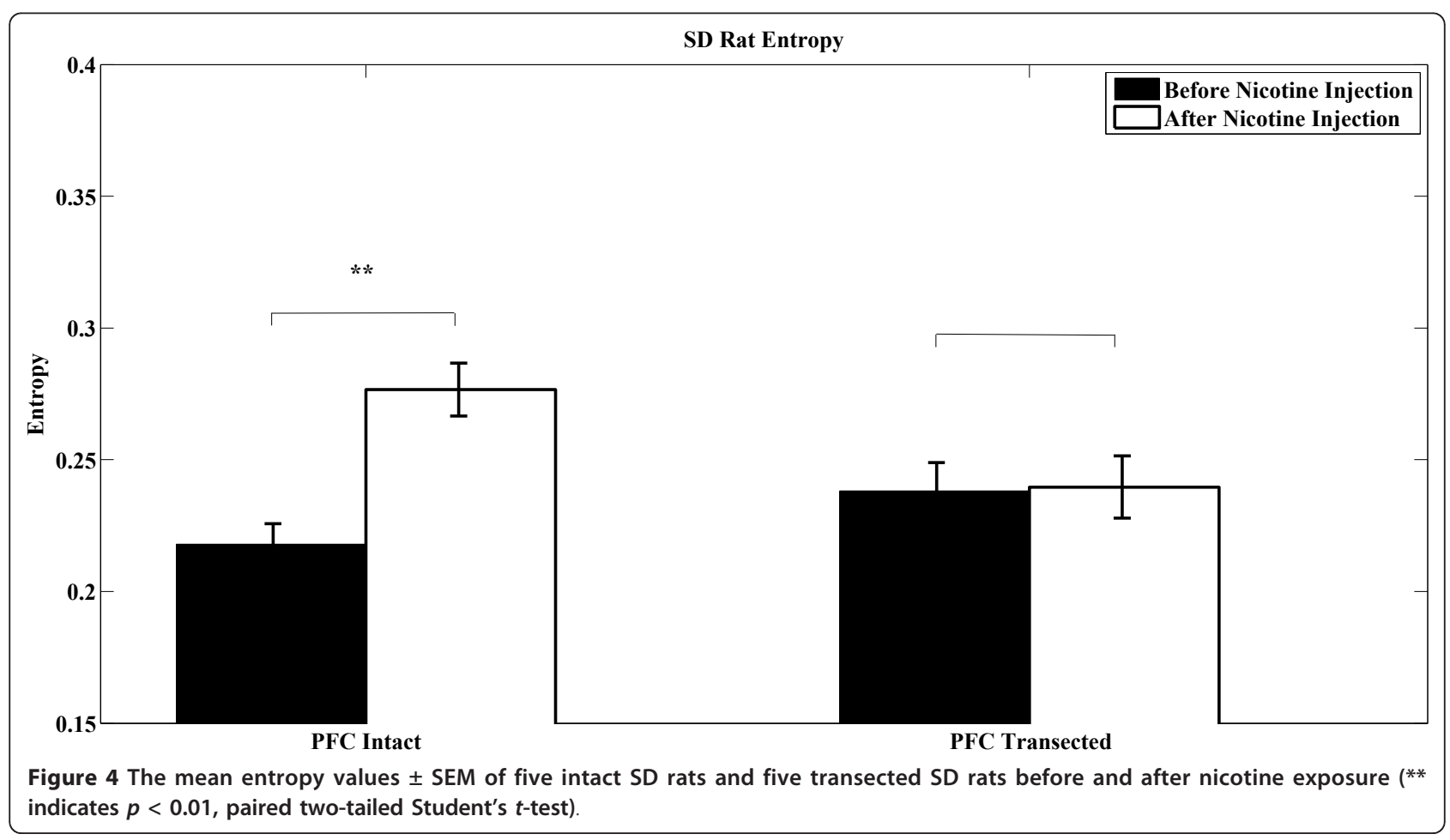


the firing of VTA DA neurons and that this effect is based on the intact input from PFC.

The increase of the excitatory drive onto the DA neurons is activated by presynaptic terminals of glutamatergic afferents induced by nicotine $[31,72,73]$. This potentiated glutamatergic drive causes DA neurons to fire more in a burst or phasic mode [30,31], since the firing rate and pattern of VTA DA neurons change with nicotine exposure. We speculate the increased complexity in PFC intact subject is due to a close functional coupling between PFC and VTA and the increased neural activity in VTA DA neurons. Our analysis demonstrated that the complexity/entropy values of neural activity after nicotine exposure were significantly increased when the connection between PFC and VTA is intact. On the other hand, the complexity/entropy values have no significant change when the input from PFC to VTA is disconnected. The reason for the increased complexity and entropy is the increased neural activity resulted from nicotine exposure.

The PFC and VTA have close functional coupling. Stimulation of PFC increases burst firing in VTA DA neurons, while deletion of PFC induces the opposite effect $[17-19,21,74]$. Gao et al [40] reported that under non-stimulation conditions, the activity of VTA DA neurons covaried with PFC neuronal activity, suggesting a close functional coupling between PFC and VTA [40]. Evidence indicates a key control of VTA neuronal function by PFC [38].

Our analysis indicates that the LZ estimators and entropy are useful tools for the characterization of the dynamical changes in VTA DA neuronal activity. As demonstrated in our analysis, such changes could be quantitatively represented as an impairment of neuronal firing during nicotine exposure and PFC transection.

\section{Acknowledgements}

We would like to thank Ms. Jessica Diefenderfer for her editing the manuscript.

\begin{abstract}
Authors' contributions
TC performed experiments and the data analysis and helped to write the manuscript, DZ helped with the experiments and helped to write the manuscript, $A D$ contributed to the data analysis and helped to write the manuscript, YMA helped with the experiments and helped to write the paper. MA oversaw the data collection, the data analysis, and helped to write the manuscript. All authors read and approved the final manuscript.
\end{abstract}

\section{Competing interests}

The authors declare that they have no competing interests.

Received: 13 July 2010 Accepted: 27 February 2011

Published: 27 February 2011

\section{References}

1. Kauer JA, Malenka RC: Synaptic plasticity and addiction. Nature Reviews Neuroscience 2007, 8:844-858.

2. Kalivas PW: Interactions between dopamine and excitatory amino acids in behavioral sensitization to psychostimulants. Drug and Alcohol Dependence 1995, 37:95-100.
3. Tong ZY, Overton PG, Martinez-Cue C, Clark D: Do non-dopaminergic neurons in the ventral tegmental area play a role in the responses elicited in A10 dopaminergic neurons by electrical stimulation of the prefrontal cortex? Exp Brain Res 1998, 118:466-476.

4. Wolf ME: The role of excitatory amino acids in behavioral sensitization to psychomotor stimulants. Prog Neurobiol 1998, 54:679-720.

5. Berke JD, Hyman SE: Addiction, dopamine, and the molecular mechanisms of memory. Neuron 2000, 25:515-532.

6. Corrigall WA, Franklin KB, Cohen KM, Clarke PB: The mesolimbic dopaminergic system is implicated in the reinforcing effects of nicotine. Psychopharmacology (Berl) 1992, 107:285-289.

7. Picciotto MR, Corrigall WA: Neuronal systems underlying behaviors related to nicotine addiction: neural circuits and molecular genetics. J Neurosci 2002, 22:3338-3341.

8. Watkins SS, Koob GF, Markou A: Neural mechanisms underlying nicotine addiction: acute positive reinforcement and withdrawal. Nicotine Tob Res 2000, 2:19-37.

9. Wang F, Chen $\mathrm{H}$, Steketee JD, Sharp BM: Upregulation of ionotropic glutamate receptor subunits within specific mesocorticolimbic regions during chronic nicotine self-administration. Neuropsychopharmacology 2007, 32:103-109.

10. Kalivas PW: Neurotransmitter regulation of dopamine neurons in the ventral tegmental area. Brain Res Rev 1993, 18:75-113.

11. White FJ: Synaptic regulation of mesocorticolimbic dopamine neurons. Annu Rev Neurosci 1996, 19:405-436.

12. Overton $P G$, Clark D: Burst firing in midbrain dopaminergic neurons. Brain Res Rev 1997, 25:312-334

13. Dong Y, Nasif FJ, Tsui JJ, Ju WY, Cooper DC, Hu XT, Malenka RC, White FJ: Cocaine-induced plasticity of intrinsic membrane properties in prefrontal cortex pyramidal neurons: adaptations in potassium currents. I Neurosci 2005, 25:936-940.

14. Gao C, Wolf M: Dopamine alters AMPA receptor synaptic expression and subunit composition in dopamine neurons of the ventral tegmental area cultured with prefrontal cortex neurons. J Neurosci 2007, 27:14275-14285.

15. Miller EK, Cohen JD: An integrative theory of prefrontal cortex function. Annu Reve Neurosci 2001, 24:167-202.

16. Miller EK, Freedman DJ, Wallis JD: The prefrontal cortex: categories, concepts and cognition. Philos Trans R Soc Lond B Biol Sci 2002, 357:1123-1136.

17. Gariano RF, Groves PB: Burst firing induced in midbrain dopamine neurons by stimulation of the medial prefrontal and anterior cingulated cortices. Brain Res 1988, 462:194-198.

18. Svensson TH, Tung CS: Local cooling of pre-frontal cortex induces pacemaker-like firing of dopamine neurons in rat ventral tegmental area in vivo. Acta Physiol Scand 1989, 136:135-136.

19. Murase S, Grenhoff J, Chouvet G, Gonon FG, Svensson TH: Prefrontal cortex regulates burst firing and transmitter release in rat mesolimbic dopamine neurons studied in vivo. Neurosci Lett 1993, 157:53-56.

20. Overton PG, Tong ZY, Clark D: A pharmacological analysis of the burst events induced in midbrain dopaminergic neurons by electrical stimulation of the prefrontal cortex in the rat. J Neural Transm 1996, 103:523-540.

21. Tong $Z Y$, Overton $P G$, Clark D: Stimulation of the prefrontal cortex in the rat induces patterns of activity in midbrain dopaminergic neurons which resemble natural burst events. Synapse 1996, 22:195-208.

22. Saal D, Dong Y, Bonci A, Malenka RC: Drugs of abuse and stress trigger a common synaptic adaptation in dopamine neurons. Neuron 2003, 37:577-582.

23. Robinson TE, Berridge KC: The psychology and neurobiology of addiction: an incentive-sensitization view. Addiction 2000, 95(Suppl 2):S91-117.

24. Everitt BJ, Wolf ME: Psychomotor stimulant addiction: a neural systems perspective. J Neurosci 2002, 22:3312-3320.

25. Li Y, Vartanian AJ, White FJ, Xue XJ, Wolf ME: Effects of the AMPA receptor antagonist NBQX on the development and expression of behavioral sensitization to cocaine and amphetamine. Psychopharmacology (Berl) 1997, 134:266-276.

26. Li Y, Hu XT, Berney TG, Vartanian AJ, Stine CD, Wolf ME, White FJ: Both glutamate receptor antagonists and prefrontal cortex lesions prevent induction of cocaine sensitization and associated neuroadaptations. Synapse 1999, 34:169-180. 
27. Tzschentke TM: Pharmacology and behavioral pharmacology of the mesocortical dopamine system. Prog Neurobiol 2001, 63:241-320.

28. Shi WX, Pun CL, Zhou Y: Psychostimulants induce low-frequency oscillations in the firing activity of dopamine neurons. Neuropsychopharmacology 2004, 29:2160-2167.

29. Shi WX: Slow oscillatory firing: a major firing pattern of dopamine neurons in the ventral tegmental area. J Neurophysiol 2005, 94:3516-3522.

30. Grenhoff J, Aston-Jones G, Svensson TH: Nicotinic effects on the firing pattern of midbrain dopamine neurons. Acta Physiol Scand 1986, 128:351-358

31. Schilstrom B, Rawal N, Mameli-Engvall M, Nomikos GG, Svensson TH: Dual effects of nicotine on dopamine neurons mediated by different nicotinic receptor subtypes. Int I Neuropsychopharmacol 2003, 6:1-11.

32. Wu J, Zhang D, Lukas RJ: Impact of prefrontal cortex (PFC) in systemic nicotine-induced midbrain dopamine neuron excitation [abstract]. Program No. 34.5. 2009 Neuroscience Meeting Planner Chicago, IL: Society for Neuroscience; 2009.

33. Zhang D, Gao M, Xu D, Shi WX, Gutkin B, Lukas R, Wu J: Nicotine perturbs functional coupling between the prefrontal cortex and the ventral tegmental area in vivo: A new mechanism of nicotine reward., submitted for publication.

34. Friedman A, Friedman Y, Dremencov E, Yadid G: VTA dopamine neuron bursting is altered in an animal model of depression and corrected by desipramine. J Mol Neurosci 2008, 34:201-209.

35. Steffensen SC, Taylor SR, Horton ML, Barber EN, Lyle LT, Stobbs SH, Allison DW: Cocaine disinhibits dopamine neurons in the ventral tegmental area via use-dependent blockade of GABA neuron voltagesensitive sodium channels. Eur J Neurosci 2008, 28:2028-2040.

36. Rieke F, Warland D, Van Steveninck R, Bialek W: Spikes: Exploring the Neural Code Cambridge: MIT Press; 1999

37. Bunney BS, Walters JR, Roth RH, Aghajanian GK: Dopaminergic neurons: effect of antipsychotic drugs and amphetamine on single cell activity. $J$ Pharmacol Exp Ther 1973, 185:560-571.

38. Grace AA, Bunney BS: Intracellular and extracellular electrophysiology of nigral dopaminergic neurons - 1. Identification and characterization. Neuroscience 1983, 10:301-315.

39. Ungless MA, Magill PJ, Bolam JP: Uniform inhibition of dopamine neurons in the ventral tegmental area by aversive stimuli. Science 2004, 303:2040-2042.

40. Gao M, Liu CL, Yang S, Jin GZ, Bunney BS, Shi WX: Functional coupling between the prefrontal cortex and dopamine neurons in the ventral tegmental area. J Neurosci 2007, 27:5414-5421.

41. Gao M, Jin Y, Yang K, Zhang D, Lukas RJ, Wu J: Mechanisms involved in systemic nicotine-induced glutamatergic synaptic plasticity on dopamine neurons in the ventral tegmental area. J Neurosci 2010, 30:13814-13825.

42. Mereu G, Yoona KW, Boi V, Gessaa GL, Naesa L, Westfall TC: Preferential stimulation of ventral tegmental area dopaminergic neurons by nicotine. European Journal of Pharmacology 1987, 141:395-399.

43. Stolerman IP, Bunker P, Jarvik ME: Nicotine Tolerance in Rats: Role of Dose and Dose Interval. Psychopharmacologia (Berl) 1974, 34:317-324

44. Grenhoff J, Aston-Jones G, Svensson TH: Nicotinic effects on the firing pattern of midbrain dopamine neurons. Acta Physiologica Scandinavica 2008, 128:351-358

45. Schilström B, Fagerquist MV, Zhang X, Hertel P, Panagis G, Nomikos GG, Svensson TH: Putative role of presynaptic $a 7^{*}$ nicotinic receptors in nicotine stimulated increases of extracellular levels of glutamate and aspartate in the ventral tegmental area. Synapse 2000, 38:375-383.

46. Lempel A, Ziv J: On the complexity of finite sequences. IEEE Trans Inform Theory 1976, 22(1):75-88.

47. Hu J, Gao J, Principe J: Analysis of biomedical signals by the Lempel-Ziv complexity: the effect on finite data size. IEEE Trans Biomed Eng 2006, 53(12):2606-2609.

48. Amigo JM, Szczepanski J, Wajnryb E, Sanchez-Vives MV: Estimating the entropy rate of spike trains via Lempel-Ziv complexity. Neural Computat 2004, 16(4):717-736.

49. Aboy M, Hornero R, Abasolo D, Alvarez D: Interpretation of the Lempel-Ziv complexity measure in the context of biomedical signal analysis. IEEE Trans Biomed Eng 2000, 53(11):2282-2288.

50. Akay YM, Dragomir A, Song C, Wu J, Akay M: Hippocampal gamma oscillations in rats. IEEE Eng Med Biol Mag 2009, 28(6):92-95.
51. Kurths J, Schwarz U, Witt A, Krampe RT, Abel M: Measures of complexity in signal analysis, chaotic, fractal and nonlinear signal processing. AIP 1995, 375:33-54.

52. Kaspar F, Schuster HG: Easily calculable measure for the complexity of spatiotemporal patterns. Phys Rev 1987, 36A:842-848.

53. Tang XZ, Tracy ER, Boozer AD, DeBrauw A, Brown R: Symbol sequence statistics in noisy chaotic signal reconstruction. Chaos 1995, 51:3871-3889.

54. Radhakrishnan N, Wilson JD, Loizou P: An alternate partitioning technique to quantify the regularity of complex time series. Int I Bifurcation Chaos 2000, 10:1773-1779.

55. Zhang X, Zhu Y, ThaKor NV, Wang Z: Detecting ventricular tachycardia and fibrillation by complexity measure. IEEE Trans Biomed Eng 1984, BME31:770-778.

56. Zhang X, Roy RJ: Detecting movement during anesthesia by EEG complexity analysis. Med Biol Eng Comput 1999, 37:327-334.

57. Orlov YL, Potapov VN: Complexity: An internet resource for analysis of DNA sequence complexity. Nucleic Acids Res 2004, 32: W628-W633.

58. Gusev VD, Nemytikova LA, Chuzhanova NA: On the complexity measures of genetic sequences. Bioinformatics 1999, 15:994-999.

59. Stern L, Allison L, Coppel RL, Dix Tl: Discovering patterns in plasmodium falciparum genomic DNA. Mol Biochem Parasitol 2001, 118:175-186.

60. Szczepaski J, Amigo JM, Wajnryb E, Sanchez-Vives MV: Application of Lempel-Ziv complexity to the analysis of neural discharges. Network 2003, 14:335-350.

61. Otu HH, Sayood K: A new sequence distance measure for phylogenetic tree construction. Bioinformatics 2003, 19:2122-2130

62. Wu X, Xu J: Complexity and brain function. Acta Biophys $\operatorname{Sin} 1991$, 7:103-106.

63. Xu J, Liu Z, Liu R, Yang QF: Information transformation in human cerebral cortex. Physica D 1997, 106:363-374

64. Abasolo D, Hornero R, Gomez C, Garcia M, Lopez M: Analysis of EEG background activity in Alzheimer's disease patients with Lempel-Ziv complexity and central tendency measure. Med Eng Phys 2006, 28:315-322.

65. Radhakrishnan N, Gangadhar B: Estimating regularity in epileptic seizure time-series data. IEEE Eng Med Biol Mag 1998, 17:89-94.

66. Zhang XS, Zhu YS, Zhang XJ: New approach to studies on ECG dynamics: extraction and analysis of QRS complex irregularity time series. Med Biol Eng Comput 1997, 35:467-473.

67. Aboy M, Hornero R, Abasolo D, Alvarez D: Interpretation of the Lempel-Ziv complexity measure in the context of biomedical signals. IEEE Trans Biomed Eng 2006, 53:2282-2288.

68. Szczepaski J, Amigo JM, Wajnryb E, Sanchez-Vives MV: Characterizing spike trains with Lempel-ziv complexity. Neurocomputing 2004, 58-60:79-84.

69. Akay M, Wang K, Akay YM, Dragomir A, Wu J: Nonlinear dynamical analysis of carbachol induced hippocampal oscillations in mice. Acta Pharm Sin 2009, 30:859-867.

70. Dragomir A, Akay YM, Akay M: Modeling carbachol-induced hippocampal network synchronization using hidden markov models. J Neural Eng 2010, 7:056012.

71. Ferenets R, Lipping T, Anier A, Jantti V, Melto S, Hovilehto S: Comparison of entropy and complexity measures for the assessment of depth of sedation. IEEE Trans Biomed Eng 2006, 53:1067-1077.

72. Pidoplichko VI, Noguchi J, Areola OO, Liang Y, Peterson J, Zhang T, Dani JA: Nicotinic cholinergic synaptic mechanisms in the ventral tegmental area contribute to nicotine addiction. Learn Mem 2004, 11:60-69.

73. Mansvelder HD, McGehee DS: Long-term potentiation of excitatory inputs to brain reward areas by nicotine. Neuron 2000, 27:349-357.

74. Overton PG, Tong ZY, Brain PF, Clark D: Preferential occupation of mineralocorticoid receptors by corticosterone enhances glutamateinduced burst firing in rat midbrain dopaminergic neurons. Brain Res 1996, 737:146-154.

doi:10.1186/1743-0003-8-13

Cite this article as: Chen et al: Complexity of VTA DA neural activities in response to PFC transection in nicotine treated rats. Journal of NeuroEngineering and Rehabilitation 2011 8:13. 